\title{
A Novel Approach to Rainfall Rate Estimation based on Fusing Measurements from Terrestrial Microwave and Satellite Links
}

\author{
Fabio Saggese*, Filippo Giannetti, and Vincenzo Lottici \\ Dipartimento di Ingegneria dell'Informazione, University of Pisa, Italy
}

\begin{abstract}
Reliable, cheap and accurate measurements of rainfall rate are growing to be more and more important in many sectors as: meteorology, agriculture, flood warning, and weather forecasting. Recently, indeed, the development of novel competitive techniques has been pushed in order to improve accuracy and reliability performance, such as commercial microwave links and broadcast satellite links. The aim of the current paper is to extend previous works of the literature based on land wireless links only. The basic idea consists in synergically employing both land and satellite based approaches together, by collecting and properly fusing the corresponding measurements. To this end, an iterative optimization procedure has been developed. As shown by numerical results, the proposed procedure gives the estimated rainfall map with a considerable accuracy and improved performance respect to the conventional algorithm based on terrestrial link only.
\end{abstract}

\section{Introduction}

Accurate measurements of rainfall are growing to be more and more high-priority in meteorology, agriculture, flood warning, and weather forecasting. Atmospheric environmental monitoring currently include surface sensors, radar, and satellite systems. From one side, these solutions require non negligible installation and operating costs, to the other one, the measurements show a temporal and spatial resolution that is often non sufficient for the tasks of interest. Recently, the above requirements and constraints have pushed the development of novel competitive techniques capable of improving accuracy performance at the cost of affordable complexity. We are referring to: $i$ ) existing commercial microwave links (CMLs) to monitor and estimate rain rate on specific areas (see [1] the references therein), and $i i$ ) the measurement of the received signal level at the ground station of direct-tohome (DTH) broadcast satellite links (BSLs) [2], [3]. In the current work, we develop an extension of the algorithm proposed in [1]. The basic idea relies on properly making use of both land and satellite based approaches, i.e. the CML and BSL, in order to obtain a more accurate rainfall rate map.

Contribution. The proposed joint approach, gives remarkable appealing advantages, and so defines the innovative contributions of the work:

Efficiency: both CMLs and BSLs exploit already existing wireless infrastructure, at no extra costs for the required equipment, the installation and operating conditions.

Coverage: CML coverage can be improved by including satellite terminals already installed at domestic premises for TV reception. Additionally, more satellite devices can be purposely installed in areas not adequately covered by terrestrial microwave links, where the deployment of conventional methods of observation, as rain gauges and weather radars, is impractical. Diversity: measuring the signal levels coming from different links, terrestrial and satellite, provides a diversity gain which is the key to improve the accuracy and reliability of the overall joint system.

Accuracy: the numerical results obtained by simulations corroborate the effectiveness of the proposed mixed strategy and quantify the improvements over the conventional systems based on CMLs only.

\section{Environmental scenario}

Geometrical model. Let us consider a threedimensional scenario defined as a parallelepiped $\mathcal{P}$ with a square base having the area $A$ to be monitored. A set of Cartesian coordinates with the origin located on the center of $A$ allows to reference each point inside $\mathcal{P}$ as $\mathbf{p}=[x, y, z]^{\mathrm{T}} \in \mathcal{P}$. Thus, the coordinates are limited as $-\sqrt{A} \leq x, y \leq \sqrt{A}, \forall x, y$, and $0 \leq z \leq h_{0}, \forall z$, assuming that the $z$-axis is limited by the $0^{\circ} \mathrm{C}$ isotherm height $h_{0}$ as shown in Fig. 1, since above $h_{0}$, i.e., inside the solid layer, rain can not be formed [2]. In order to gather up and fuse together the data measurements available from both the terrestrial microwave and satellite links, let us assume that $N=N_{w}+N_{s}$ wireless links are active within $\mathcal{P}$, where $N_{w}$ and $N_{s}$ are the number of CMLs and BSLs, respectively. To be specific, each $\ell$ th link is delimited by the points $\mathbf{p}_{\ell, 1}=\left[x_{\ell, 1}, y_{\ell, 1}, z_{\ell, 1}\right]^{\mathrm{T}} \in \mathcal{P}$ and $\mathbf{p}_{\ell, 2}=\left[x_{\ell, 2}, y_{\ell, 2}, z_{\ell, 2}\right]^{\mathrm{T}} \in \mathcal{P}, \ell=1, \cdots, N$. We note that 


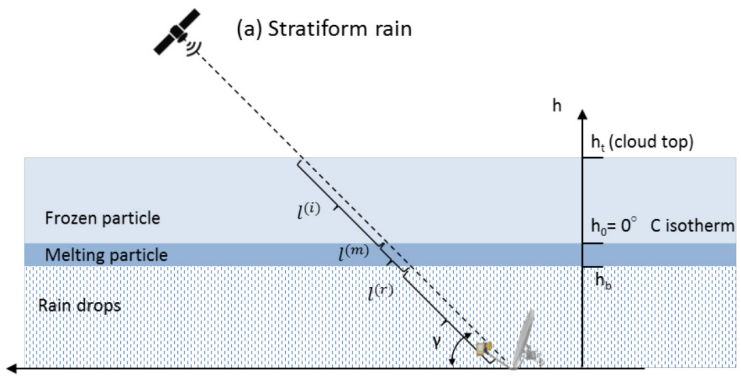

Figure 1. BSL's stratiform rain model and geometry.

for each CML we have $z_{\ell, 1}=z_{\ell, 2}$, whereas for each BSL $z_{\ell, 2}=h_{0}$. Thus, the length of each link results as

$$
L_{\ell}=\sqrt{\left(\mathbf{p}_{\ell, 1}-\mathbf{p}_{\ell, 2}\right)^{\mathrm{T}}\left(\mathbf{p}_{\ell, 1}-\mathbf{p}_{\ell, 2}\right)},
$$

$(\cdot)^{\mathrm{T}}$ denoting the transpose of a vector.

Rainfall attenuation model. In a wireless communication link working in the frequency range 10-30 $\mathrm{GHz}$, the rainfall attenuation depends (with a good approximation) on the rainfall rate according to the so-called power law formula [4]

$$
A_{\mathrm{R}}=a R^{b},
$$

where $A_{\mathrm{R}}$ is the attenuation due to the rainfall in $\mathrm{dB} / \mathrm{km}, R$ is the rainfall rate measured in $\mathrm{mm} / \mathrm{h}$. Moreover, $a$ and $b$ are coefficients depending on the frequency, polarization and the rain drop size distribution (DSD), and they can be evaluated according the ITU-R recommendation [5], applying the analytic expressions in [6], or through a non-linear regression in the case $A_{\mathrm{R}}, R$ and DSD are known [2]. Given the values of $a, b$, and $A_{\mathrm{R}}$, the rainfall rate can be evaluated as

$$
R=\alpha A_{\mathrm{R}}^{\beta}
$$

where $\alpha=a^{-\beta}$ and $\beta=b^{-1}$. It is worth noting that each wireless link suffers from an overall attenuation of which $A_{\mathrm{R}}$, due the rainfall, is only a component. Stateof-the-art methods as [2] or [7], however, have shown that the rainfall contribution can be easily extracted. Consequently, in order to estimate the rainfall rate, in our model we will focus on the estimation of the component $A_{\mathrm{R}}$ only. In addition, as far as the BSLs are concerned in a stratiform rain model, for simplicity reasons, the attenuation measured at each satellite receiver induced by the melting layer will be discarded (see Fig. 1), and therefore, we will take into account only the attenuation due to the liquid layer. ${ }^{1}$

\section{Estimation algorithm}

Map link preprocessing. In order to estimate the rainfall rate map, proper data need to be evaluated

\footnotetext{
${ }^{1}$ The approach can be generalized following the methods illustrated in [2], [8]
}

in specific points of $\mathcal{P}$. To this end, each $\ell$ th link is split in $K_{\ell}$ intervals each with length $D$, wherein the rainfall rate on the $x-y$ plane is assumed to be nearly constant. So, $K_{\ell}$ is

$$
K_{\ell}=\left\lceil L_{\ell} \cos \left(\theta_{\mathrm{el}}\right) / D\right\rceil, \quad \forall \ell,
$$

where it is worth noting that CMLs has $\theta_{\mathrm{el}}=0$, while the BSLs are characterized by the presence of the slanted path between the transmitter and receiver. Following the approach of [1], we define a data point for each interval, which is uniquely specified by the coordinates of the center of the interval itself. Hence, we denote the total number of the above data points as $M=\sum_{\ell=1}^{N} K_{\ell}$, each of them having coordinates $\mathbf{d}_{\ell, k}=\left[x_{\ell, k}, y_{\ell, k}, z_{\ell, k}\right]^{\mathrm{T}}$, and the rainfall rate value as $r_{\ell, k}, \ell \in\{1, \cdots, N\}, k \in\left\{1, \cdots, K_{\ell}\right\}$.

Point rainfall rate estimation. Let us evaluate a rainfall rate at the generic point $\mathbf{p}_{i} \in \mathcal{P}$ given $M \in \mathbb{N}_{+}$ rainfall rates corresponding to the points $\mathbf{p}_{m} \in \mathcal{P}, m=$ $1, \ldots, M$. In line with the model used in [1], we can write

$$
\mathbf{r}=\mathbf{1} \hat{r}_{i}+\boldsymbol{\nu}_{i}
$$

where $\mathbf{r} \in \mathbb{R}_{+}^{M}$ collects the known $M$ rainfall rates, $\mathbf{1} \in$ $\mathbb{R}_{+}^{M}$ is a vector of ones, $\hat{r}_{i}$ is the value to be estimated and $\boldsymbol{\nu}_{i} \in \mathbb{R}_{+}^{M}$ represents the error between the entries of $\mathbf{r}$ and $\hat{r}_{i}$, which is assumed to be jointly Gaussian, i.e. $\nu_{i} \sim \mathcal{N}\left(0, \Lambda_{i}\right)$, with $\Lambda_{i}^{-1}=\operatorname{diag}\left\{W_{i, m}\right\}, m=1, \ldots, M$. The weights $W_{i, m}$ depend on the inverse of the distance between the points $m$ and $i$, according to the Shepard's inverse distance weighting (IDW) method [9]. More specifically, such weights are evaluated as

$$
W_{i, m}=\left(\frac{\left(1-d_{i, m} / \Gamma_{i}\right)^{2}}{\left(d_{i, m} / \Gamma_{i}\right)^{2}}\right)^{+},
$$

where $(x)^{+}=\max (0, x)$, the radius of influence $\Gamma_{i}$ specify which data points play a role to estimate the rainfall rate at the generic point $i$, whereas $d_{i, m}$ denotes the distance between the $i$ th and $m$ th points. ${ }^{2}$ Concerning the BSLs, it worth observing that the satellite transmitted signals travel through $\mathcal{P}$, i.e., $0 \leq z \leq h_{0}$, while experiencing different environmental conditions, such as pressure, humidity, temperature and so on. Consequentely, when choosing the data points which are more influent in the evaluation of the rainfall rate of the $i$ th point, we combine BSL data points belonging to the same plane at a given height. This is equivalent to say that the distance $d_{i, m}$ between the $i$ th and $m$ th points is evaluated on the $x-y$ plane only as

$$
d_{i, m}=\sqrt{\left(x_{i}-x_{m}\right)^{2}+\left(y_{i}-y_{m}\right)^{2}}
$$

Hence, putting together the above facts, adopting a least-square (LS) solution yields [10]

$$
\hat{r}_{i}=\left(\mathbf{1}^{\mathrm{T}} \Lambda_{i}^{-1} \mathbf{1}\right)^{-1} \mathbf{1}^{\mathrm{T}} \Lambda_{i}^{-1} \mathbf{r} .
$$

\footnotetext{
${ }^{2}$ An equivalent yet heuristic method consists in setting $\Gamma_{i}$ as the minimum radius which includes at least 5 data points [1], [9]
} 
Optimization algorithm. Exploiting the model defined in Sect. 2 and the point rainfall rate estimation discussed above, this section formalize an iterative method to obtain the rainfall rate for the data points $r_{\ell, k}, \ell \in\{1, \cdots, N\}, k \in\left\{1, \cdots, K_{\ell}\right\}$, by essentially combining the LS estimation problem with a constrained optimization problem (OP). As example, let us start by considering the estimation of the data points of the eth link. According to [1], firstly the rainfall rates $\hat{r}_{\ell, k}$ are LS estimated through IDW from the data points of all the other links, indexed by $n \in\{1, \cdots, N \mid n \neq \ell\}$, $k=1, \cdots, K_{n}$. Then, the minimization of the distance between the rain rates $r_{\ell, k}$ and $\hat{r}_{\ell, k}$ is applied, $k=1, \cdots, K_{\ell}$. This must take into account the overall rainfall attenuation $A_{\mathrm{R} \ell}$ measured by the link $\ell$. Therefore, we assume that

$$
A_{\mathrm{R} \ell}=\sum_{k=1}^{K_{\ell}} A_{\mathrm{R} \ell, k}
$$

i.e., the total rain attenuation on the $\ell$ th $\operatorname{link} A_{\mathrm{R} \ell}$ is the sum of the rain attenuation of the $K_{\ell}$ segments. Hence, using (2), equation (9) becomes

$$
K_{\ell} R_{\ell}^{b}=\sum_{k=1}^{K_{\ell}} r_{\ell, k}^{b}, \quad \ell=1, \cdots, N,
$$

where $R_{\ell}$ is the total rainfall rate measured at the end of the $\ell$ th link. Upon denoting $\mathbf{r}_{\ell}=\left[r_{\ell, 1}, \cdots, r_{\ell, K_{\ell}}\right]^{\mathrm{T}}$ and $\hat{\mathbf{r}}_{\ell}=\left[\hat{r}_{\ell, 1}, \cdots, \hat{r}_{\ell, K_{\ell}}\right]^{\mathrm{T}}$, the optimization problem can be formalized as

$$
\underset{\mathbf{r}_{l}}{\arg \min }\left\{\left\|\mathbf{r}_{\ell}-\hat{\mathbf{r}}_{\ell}\right\|^{2} \mid K_{\ell} R_{\ell}^{b}-\sum_{k=1}^{K_{\ell}} r_{\ell, k}^{b}=0\right\} .
$$

We note that the OP (11) is not convex since the equality constraint is not affine, and as such, the optimal solution is not unique. Thus, our approach uses gradient descent based method to converge at least to a local optimal point [11]. The algorithm pseudocode is outlined in Algorithm 1, where it worth emphasizing that the rainfall rates $r_{\ell, k}$ are initialized as $r_{\ell, k}=R_{\ell}, \ell=1, \cdots, N ; k=1, \cdots, K_{\ell}$ and that the procedure is iterated until the norm of difference between two consecutive data points' rainfall rate is below a given threshold $\epsilon$.

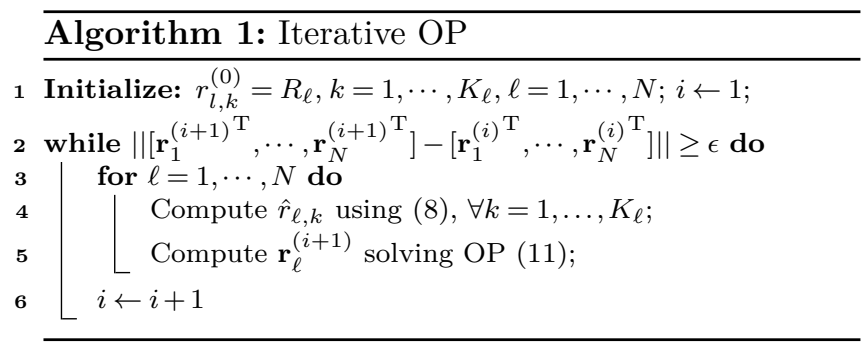

Rainfall rate mapping. After the rainfall rate of all the data points are evaluated applying the iterative OP, the rainfall rate map must be be compute.
To this aim, the area of interest $A$ is sampled in $J^{2}$ pixels, i.e., $J$ equally-spaced pixels for each side. We identify them as the grid points $\mathbf{g}_{j}, 1 \leq j \leq J^{2}$, each with $\mathbf{g}_{j}=\left[x_{j}, y_{j}, z_{j}\right]^{\mathrm{T}}$ and estimated rain rate $\hat{r}_{j}^{(g)}$. The rainfall rate map can be evaluated by applying the LS procedure (8) between the estimated rainfall rates of data points and those on the grid points.

\section{Numerical results}

In order to corroborate the effectiveness of our approach, we carried out many simulation runs for the scenario of interest. To be specific, we define a number of CMLs and BSLs, given by their location inside the area $A$ to be monitored, working frequency $f_{0}$ and polarization. The coefficient $a$ and $b$ are then computed according to [6]. The rain is simulated using a virtual cylinder of rain with constant rain rate $r$ and radius $\gamma_{\text {cyl }}$. At each simulation run, the cylinder of rain is located in a random position. The simulation parameters are shown in Table 1.

\begin{tabular}{cc|cc}
\hline$A$ & $100 \mathrm{~km}^{2}$ & $f_{0}$ & $18 \mathrm{GHz}$ \\
$J$ & 64 & polariz. & vertical \\
$D$ & $50 \mathrm{~m}$ & $a$ & 0.0601 \\
$h_{0}$ & $1 \mathrm{~km}$ & $b$ & 1.1154 \\
$\gamma_{\text {cyl }}$ & $1.5 \mathrm{~km}$ & $r$ & $10 \mathrm{~mm} / \mathrm{h}$ \\
\hline
\end{tabular}

Table 1. Simulation parameters

Figure 2 gives an example of the grid map on the $x-y$ view (first column) and its estimation (second column) for two different cases: (a) and (b). Along with the cylinder of rain (in red), 14 CMLs are positioned following a portion of the Israeli map of commercial wireless links for both cases, while in case (b), other 8 BSLs are randomly located. From the estimated maps, we can deduce that the proposed mixed approach evaluates the rainfall rate with good accuracy, and it can strongly improve the estimation respect to the conventional case using only CMLs. In the following, we present the aggregated results obtained running hundreds of simulations. The overall accuracy performance of the estimation algorithm is quantified both as the root mean square error RMSE $=\sqrt{\|\hat{\mathbf{r}}-\mathbf{r}\|^{2} / J^{2}}$, where $\mathbf{r}$ and $\hat{\mathbf{r}}$ are the real and the estimated rainfall rate maps, respectively, and as the correlation coefficient $\rho=\operatorname{cov}(\hat{\mathbf{r}}, \mathbf{r}) /\left(\sigma_{\hat{r}} \sigma_{r}\right)$, which evaluates the similarity between the actual and the estimated maps. Figures 3 and 4 depict the RMSE (solid lines) and $\rho$ (dashed lines) for near-to-the-ground height and different scenarios as a function of the number of CMLs and BSLs, respectively. It is apparent that: $i$ ) from Fig. 3 even a small number of satellites can significantly contribute to reduce the error; $i i)$ from Fig. 4 the impact on the accuracy performance of the number of CMLs is much lower respect to the number of BSLs; iii) the 

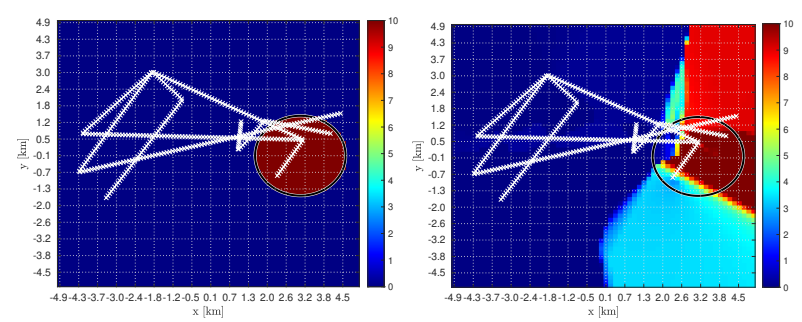

(a) $\mathrm{CML}=14, \mathrm{BSL}=0$
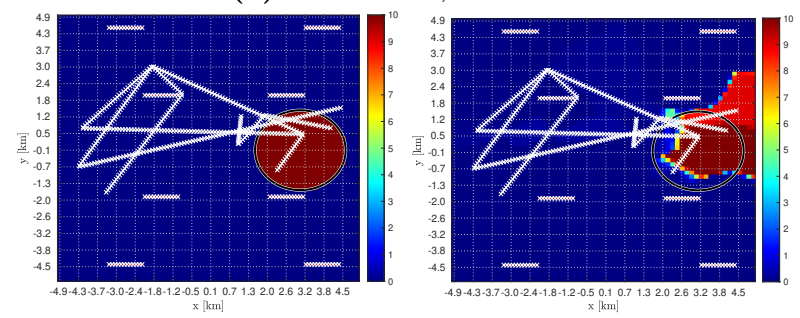

(b) $\mathrm{CML}=14, \mathrm{BSL}=8$

Figure 2. Snapshot of a simulation.

latter are easier and cheaper to put in place respect to the installation of new CMLs.

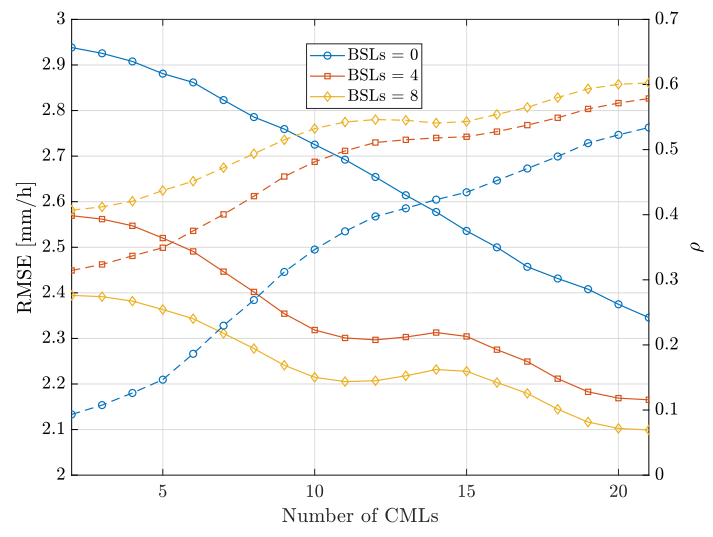

Figure 3. RMSE and $\rho$ vs. number of CMLs.

\section{Conclusion and Future Works}

In this paper, the problem of rainfall rate estimation through mixed data coming from commercial microwave links and broadcast satellites link has been studied. The simulation results show the effectiveness of the mixed approach respect to the conventional algorithms which exploit the terrestrial link only. Future works will further investigate the dependence on the environmental condition of the rain among the $z$-axis in order to refine both model and the algorithm.

\section{Acknowledgement}

Authors are indebted to Prof. Hagit Messer and Dr. Adam Eshel of Tel Aviv University (Israel) for many fruitful discussions on this topic. This paper was inspired by SVI.I.C.T.PRECIP. project, funded by FAR-

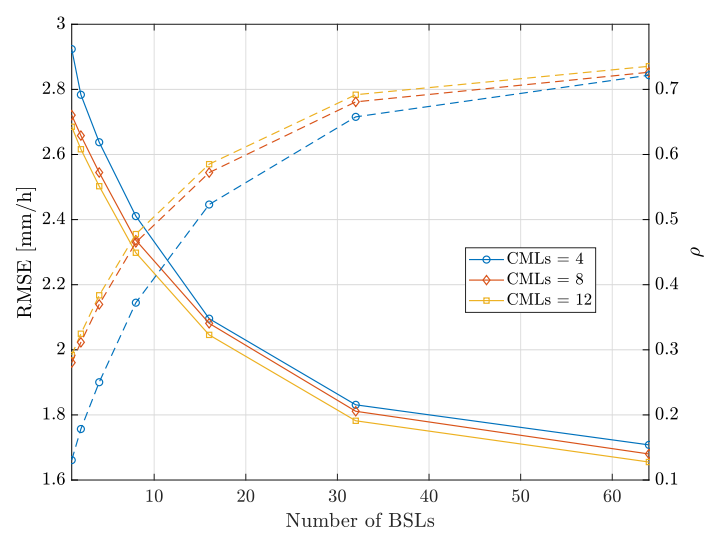

Figure 4. RMSE and $\rho$ vs. number of BSLs.

FAS 2014 program of the Tuscany Region, Italy, under agreement No. 4421.02102014.072000064, and it was supported by MISSISSIPPI project of the University of Pisa, D.R. 1528, 13-09-18, and D.R. 1937, 06-11-18.

\section{References}

[1] O. Goldshtein, H. Messer, and A. Zinevich, "Rain rate estimation using measurements from commercial telecommunications links," IEEE Trans. Signal Process., vol. 57, pp. 1616-1625, May 2009.

[2] F. Giannetti, et alii, "Real-time rain rate evaluation via satellite downlink signal attenuation measurement," Sensors, vol. 17, pp. 1864-1887, Aug. 2017.

[3] A. Gharanjik, K.V. Mishra, B.S. Mysore, B. Ottersten, "Learning-based rainfall estimation via communication satellite links," IEEE Statistical Signal Process. Workshop, freiburg, Germany, June 2018.

[4] R. Olsen, D. Rogers, and D. Hodge, "The $\mathrm{aR}^{b}$ relation in the calculation of rain attenuation," IEEE Trans. Antennas and Propag., vol. 26, no. 2, pp. 318-329, March 1978.

[5] Recommendation ITU-R, P.838-3, "Specific attenuation model for rain for use in prediction method," 1997.

[6] Z.-W. Zhao, M.-G. Zhang, and Z.-S. Wu, "Analytic specific attenuation model for rain for use in prediction methods," International Journal of Infrared and Millimeter Waves, vol. 22, no. 1, pp. 113-120, Jan. 2001.

[7] A. Zinevich, H. Messer, and P. Alpert, "Prediction of rainfall intensity measurement errors using commercial microwave communication links," Atmospheric Measurement Techniques, vol. 3, Oct. 2010.

[8] F. Giannetti, M. Moretti, R. Reggiannini, and A. Vaccaro, "The NEFOCAST system for detection and estimation of rainfall fields by the opportunistic use of broadcast satellite signals," IEEE Aerosp. Electron. Syst. Mag., June 2019.

[9] A. Eshel, J. Ostrometzky, S. Gat, P. Alpert, and H. Messer, "Spatial reconstruction of rain fields from wireless telecommunication networks-scenario-dependent analysis of IDWbased algorithms," IEEE Geosci. Remote Sens. Lett., pp. $1-5$, Sept. 2019.

[10] S. M. Kay, Statistical Signal Processing. New Jersey: Prentice Hall, 1993.

[11] S. Boyd and L. Vandenberghe, Convex Optimization. Cambridge University Press, 2004. 